\title{
Co-ingestion of antioxidant drinks with an unhealthy challenge meal fails to prevent post-prandial endothelial dysfunction: an open-label, crossover study in healthy older adults
}

\author{
K. Goszcz, D. J. Muggeridge, A. Treweeke, J. Adamson, K. Hickson and I. L. Megson \\ Free Radical Research Facility and Active Health Exercise Laboratory, Division of Biomedical Sciences, Institute of \\ Health Research and Innovation, University of the Highlands and Islands, Inverness, IV2 3JH.
}

Endothelial function is impaired for several hours after an unhealthy challenge meal (e.g high calorie, high fat) ${ }^{(1)}$. This impairment has been shown to be abolished in the presence of antioxidants ${ }^{(2)}$, suggesting that reactive oxygen species (ROS) may play an important role in the induced dysfunction. In this study, we assessed whether drinks with high-antioxidant capacity impacted endothelial dysfunction induced by an unhealthy challenge meal.

The study was an open-label randomised crossover design: 7 healthy older adults (1 male, 6 females; BMI 25-35; mean age 57 years) were recruited to the study and completed four experimental trials. Participants received either water, orange juice, green tea or red wine with a high calorie meal (>900 kcal; $>50 \mathrm{~g}$ fat) following an overnight fast. Endothelial function (primary measure) was assessed by flow-mediated dilatation (FMD) of the brachial artery immediately before (baseline) and two hours after the meal; blood samples were also taken at these time points for routine lipid and glucose analysis, as well as measurement of oxidised low density lipoprotein (ox-LDL) and oxygen radical antioxidant capacity (ORAC). Participants returned at weekly intervals to complete the remaining arms of the study. Data was analysed using a 2-factor repeated measures ANOVA.

The results demonstrate that two hours following an unhealthy meal challenge there was a substantial increase in circulating triglycerides $(\geq 100 \%, \mathrm{P}<0.001)$, but not total cholesterol or glucose (both $\mathrm{P}>0.05$ ). FMD was reduced by $\sim 30 \%$ at this timepoint, but the effect was not attenuated by co-ingestion of any of the antioxidant drinks (Fig. 1). In addition, there was no effect of the meal or condition on circulating levels of ox-LDL or ORAC $(\mathrm{P}>0.05)$.

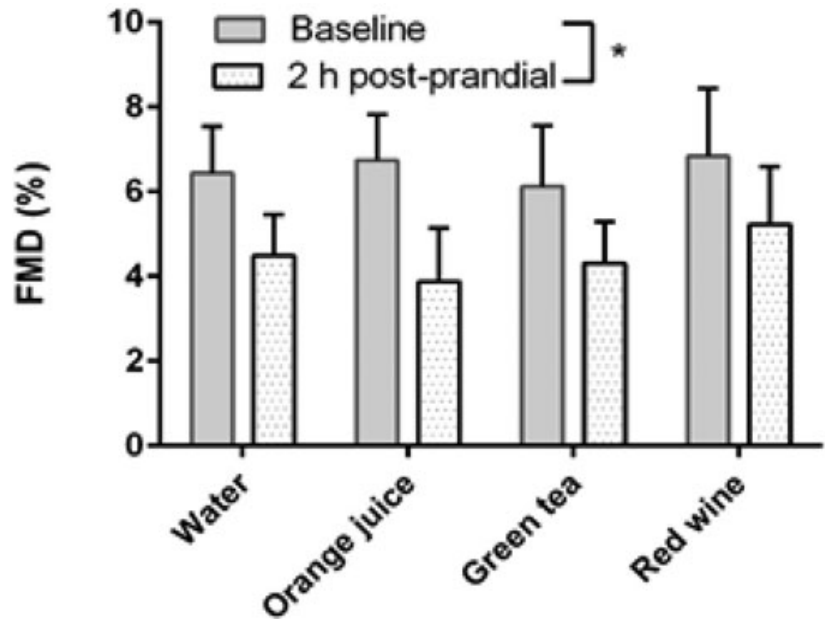

Fig. 1. Group mean + SEM FMD responses to an unhealthy challenge meal. FMD was significantly attenuated after the meal challenge (P $<0.01)$, but there was no significant difference between FMD in any of the study arms compared to the water (control) arm.

We conclude that co-ingestion of any of our test antioxidant drinks failed to protect against the substantial post-prandial endothelial dysfunction induced by an unhealthy meal challenge. That circulating ox-LDL was not influenced by the antioxidant drinks might point to either a lack of role for oxidative stress in the endothelial dysfunction experienced, or a need for complex and lengthy intracellular signalling to achieve antioxidant benefits.

This study was part funded by BBC studios. KG is funded by Highlands and Islands Enterprise; DM is supported by the European Union's INTERREG VA Programme, managed by the Special EU Programmes Body (SEUPB).

1. de Koning EJP, \& Rabelink TJ (2002) Atherosclerosis Supplements 3,11-16.

2. Beckman JA, Goldfine AB, Gordon MB \& Creager MA (2001) Circulation 103, 1618-1623. 